\title{
LOCALLY FINITE-DIMENSIONAL ALGEBRAS
}

\author{
DANIEL R. FARKAS AND ROBERT L. SNIDER ${ }^{1}$
}

\begin{abstract}
Von Neumann regularity of some locally finite-dimensional algebras is studied.
\end{abstract}

C. Lanski has asked [3] whether every simple algebraic algebra over a field is von Neumann regular. Along the same lines, Handelman asks whether a regular locally finite-dimensional algebra is locally semisimple. In this note we answer Lanski's question in the negative and discuss some of the subtleties of the second problem.

1. An example. Assume $R$ is any ring with 1 . Let $T(R)$ denote the ring $R[x] /\left(x^{2}\right)$, the polynomial ring over $R$ modulo the ideal generated by $x^{2}$. We denote the image of $x$ in $T(R)$ by $\xi$. We remind the reader of the universal property for this construction: a ring homomorphism from $T(R)$ to $S$ is completely specified by a homomorphism $R \rightarrow S$ and an assignment $\xi \mapsto \sigma$ where $\sigma \in S$ has square zero and centralizes the image of $R$.

We are interested in the case $R=M_{n}(k)$, the full ring of $n \times n$ matrices over the field $k$. (Extending the earlier notation, $\xi_{n}$ will denote the image of $x$ in $T\left(M_{n}(k)\right)$.) Since this choice of $R$ is a simple ring, it is easy to check that the only two-sided ideals of $T(R)$ are zero, the entire ring and $T(R) \xi_{n}$.

Again for $R=M_{n}(k)$ we can define a map $\varphi_{n}: T(R) \rightarrow T\left(R \otimes_{k} R\right)$ by sending $r$ in $R$ to $r \otimes 1$ and by sending $\xi_{n}$ to $\left(1 \otimes e_{1 n}\right)+\left(1 \otimes b_{n}\right) \xi_{n^{2}}$. Here $e_{1 n}$ is the usual matrix unit and $b_{n}$ is the $n \times n$ matrix exhibiting the similarity of $e_{1 n}$ and $-e_{1 n}$ : $b_{n}\left(e_{1 n}\right) b_{n}^{-1}=-e_{1 n}$.

Lemma. Suppose $\varphi_{n}: T(R) \rightarrow T\left(R \otimes_{k} R\right)$ is as above, and $\varphi_{n}\left(u+v \xi_{n}\right)=$ $u^{\prime}+v^{\prime} \xi_{n^{2}}$

(i) If $v$ is invertible so is $v^{\prime}$.

(ii) $\operatorname{rk}\left(u^{\prime}\right) \leqslant(\operatorname{rk}(u)+1) n$.

PRoOF. $u^{\prime}+v^{\prime} \xi_{n^{2}}=\left(u \otimes 1+v \otimes e_{1 n}\right)+\left(v \otimes b_{n}\right) \xi_{n^{2}}$. Since $b_{n}$ is invertible, $v \otimes$ $b_{n}$ is invertible if and only if $v$ is.

$$
\mathrm{rk}\left(u \otimes 1+v \otimes e_{1 n}\right)<\mathrm{rk}(u \otimes 1)+\operatorname{rk}\left(v \otimes e_{1 n}\right)<\operatorname{rk}(u) \cdot n+n \cdot 1
$$

Proposition. Let $\mathcal{T}$ be the direct limit

$$
T\left(M_{4}(k)\right) \stackrel{\varphi}{\rightarrow} T\left(M_{4^{2}}(k)\right) \stackrel{\varphi}{\rightarrow} T\left(M_{4^{4}}(k)\right) \stackrel{\varphi}{\rightarrow} \ldots
$$

Received by the editors March 28, 1980.

1980 Mathematics Subject Classification. Primary 16A30.

${ }^{1}$ The work of both authors was partially supported by the National Science Foundation. 
Then $\mathcal{T}$ is a locally finite-dimensional simple algebra and there is no $w \in \mathcal{T}$ such that $z w z=z$ for $z$ the image of $\xi_{4}$ in $丁$.

Proof. Write $f(n)=2^{2^{n}}$ and notice that $f(n)^{2}=f(n+1)$. The direct limit is built up from $T\left(M_{f(n)}(k)\right) \stackrel{\varphi_{f(n)}}{\rightarrow} T\left(M_{f(n+1)}(k)\right)$. Let $u_{f(n)}+v_{f(n)} \xi_{f(n)}$ be the image of $\xi_{4}$ in $M_{f(n)}(k)$. We estimate the rank of $u_{n}$ by applying induction to the lemma:

$$
\operatorname{rk}\left(u_{f(n)}\right)<(f(n)-4) / 3
$$

Now it easily follows that $\operatorname{rk}\left(u_{f(n)}\right)<f(n) / 2$.

If $z w z=z$ in the limit $\mathcal{T}$ then a similar equality holds at some stage. There is an $x+y \xi_{f(n)} \in T\left(M_{f(n)}(k)\right)$ such that

$$
\left(u_{f(n)}+v_{f(n)} \xi\right)(x+y \xi)\left(u_{f(n)}+v_{f(n)} \xi\right)=u_{f(n)}+v_{f(n)} \xi .
$$

Comparing coefficients of $\xi$ (and temporarily dropping some subscripts to relieve the clutter), $u_{f(n)} x v_{f(n)}+v_{f(n)} x u_{f(n)}+u_{f(n)} y u_{f(n)}=v_{f(n)}$. Since $v_{f(n)}$ is invertible,

$$
\begin{aligned}
f(n) & =\operatorname{rk}\left(v_{f(n)}\right)=\operatorname{rk}\left(u_{f(n)} \cdot(x v+y u)+(v x) u_{f(n)}\right) \\
& \leqslant 2 \operatorname{rk}\left(u_{f(n)}\right)<f(n),
\end{aligned}
$$

a contradiction.

The nature of a direct limit immediately implies that $\mathcal{T}$ is locally finitedimensional. As to simplicity, each nonzero element of $\mathcal{T}$ has a nonzero ancestor $c+d \xi_{\nu}$ in some $T\left(M_{\nu}(k)\right)$. We have already remarked that if the ideal generated by $c+d \xi_{p}$ in $T\left(M_{\nu}(k)\right)$ does not contain 1 then $c=0$ and this ideal must be generated by $\xi_{v}$. Replace the original element in $\mathcal{T}$ with the image of $\xi_{v}$. This element is also the image of $\varphi_{\nu}\left(\xi_{\nu}\right)=1 \otimes e_{1 \nu}+\left(1 \otimes b_{\nu}\right) \xi_{\nu}$ and the ideal generated by it in $T\left(M_{v^{2}}(k)\right)$ contains 1 .

Observe that the example is a locally finite, countable dimensional, simple algebra which is not a direct limit of semisimple Artinian subalgebras.

2. Regular algebras. The authors tried to construct a von Neumann regular, locally finite-dimensional algebra which is not locally semisimple by modifying the procedure in the first section. Contrary to our expectations, these specially constructed algebras are indeed locally semisimple. More precisely, any direct limit $\lim _{j} M_{\tau(j)}(k)[x] /\left(x^{\sigma(j)}\right)$ which is regular, is also locally semisimple.

This is established by extending a calculation of Levitzki [5].

THEOREM. A nilpotent element in a von Neumann regular $k$-algebra $R$ is contained in a subalgebra isomorphic to a finite product of matrix algebras over $k$.

Proof. Say $a^{n}=0, a^{n-1} \neq 0$ for some $a \in R$. We argue by induction on $n$. By regularity, we can find a $b \in R$ such that $a^{n-1} b a^{n-1}=a^{n-1}$ and $b a^{n-1} b=b$.

For $1 \leqslant i, j \leqslant n$ define $e_{i j}=a^{n-i} b a^{j-1}, E_{t}=e_{t t} \circ e_{t+1, t+1} \circ \cdots \circ e_{n n}$ and $E_{n+1}$ $=0 ; f_{i j}=e_{i j}\left(1-E_{j+1}\right)\left(\right.$ here $\left.e \circ e^{\prime}=e+e^{\prime}-e e^{\prime}\right)$. Observe

(i) $E_{t} \in R a^{t-1}$.

(ii)

$$
a f_{i j}=\left\{\begin{array}{l}
0 \text { for } i=1 \\
f_{i-1, j} \text { for } i>1
\end{array}\right.
$$


Indeed, $a e_{i j}=a^{n-(i-1)} b a^{j-1}$.

(iii)

$$
f_{i j} a=\left\{\begin{array}{l}
0 \text { for } j=n, \\
f_{i, j+1} \text { for } j<n .
\end{array}\right.
$$

If $i, j>1, a e_{i j}=e_{i-1, j}=e_{i-1, j-1} a$, so $E_{t} a=a E_{t+1}$ for $t=1, \ldots, n$. Thus $f_{i j} a=$ $e_{i j}\left(1-E_{j+1}\right) a=e_{i j} a\left(1-E_{j+2}\right)=e_{i, j+1}\left(1-E_{j+2}\right)=f_{i, j+1}$ for $j<n$.

(iv) Set $f=f_{11}+f_{22}+\cdots+f_{n n}$. Then af $=f a=\sum_{j>1} f_{j-1, j}$.

Apply (ii) and (iii).

(v) $a^{n-1} f=a^{n-1}$.

By (ii), $a^{n-1} f=f_{1 n}=e_{1 n}=a^{n-1} b a^{n-1}=a^{n-1}$.

We next claim that the $f_{i j}$ are matrix units. Calculating,

$$
f_{i j} f_{j k}=e_{i j}\left(1-E_{j+1}\right) e_{j k}\left(1-E_{k+1}\right) \text {. }
$$

$\operatorname{But}\left(E_{j+1}\right) e_{j k} \in R a^{j} a^{n-j} b a^{k-1}=0$, so

$$
\begin{aligned}
f_{i j} f_{j k} & =e_{i j} e_{j k}\left(1-E_{k+1}\right)=a^{n-i} b a^{n-1} b a^{k-1}\left(1-E_{k+1}\right) \\
& =a^{n-i} b a^{k-1}\left(1-E_{k+1}\right)=f_{i k} .
\end{aligned}
$$

Since $f_{i j} f_{k l}=f_{i j} f_{j j} f_{k k} f_{k l}$, we will be done once we show $f_{j j} f_{k k}=0$ for $j \neq k$. If $j>k$ then $j+n-k>n$ implies $E_{j+1} e_{k k} \in R a^{j} a^{n-k} R=0$. Thus $f_{j j} f_{k k}=$ $e_{i j} e_{k k}\left(1-E_{k+1}\right)=0$. On the other hand, if $j<k$ then a result on page 49 of [4] states that $E_{j+1} e_{k k}=e_{k k}$. Thus $f_{j j} f_{k k}=e_{j j}\left(e_{k k}-e_{k k}\right)\left(1-E_{k+1}\right)=0$.

Finally consider the matrix algebra spanned by the $f_{i j}$. The element $a f$ is inside, by (iv). According to (v), $(1-f) a^{n-1}=0$; successive application of (iv) yields $\{(1-f) a(1-f)\}^{n-1}=0$. By induction, $(1-f) a(1-f)$ is contained in an appropriate semisimple subalgebra of $(1-f) R(1-f)$. We put together the pieces by observing that $a=a f+(1-f) a(1-f)$.

Notice that there is no problem in enlarging the semisimple algebra to contain 1 if we insist that subalgebras share the identity element with the entire algebra.

We return to the direct limit mentioned at the beginning of this section. Local semisimplicity follows from the

COROLlaRY. If $S \cong M_{n}(k)[x] /\left(x^{m}\right)$ is a subring (with the same 1) of the von Neumann regular $k$-algebra $R$ then $S$ is contained in a finite-dimensional semisimple subalgebra of $R$.

Proof. Write $S=A+A \xi$ where $A \cong M_{n}(k)$ and $\xi$ is the image of $x$ in $S$. Certainly $\xi$ centralizes $A$. It is well known [4] that $R=A \otimes_{k} C\left(\cong M_{n}(C)\right)$ where $C$ is the centralizer of $A$ in $R$. A straightforward manipulation with matrix units shows that $C \cdot 1$ is a $C$-C -bimodule summand of $M_{n}(C)$ and that, as a result, the regularity of $M_{n}(C)$ forces $C$ to be regular. Apply the theorem to $\xi$ in $C$ : There is a finite-dimensional subalgebra $B$ of $C$ which contains $\xi$ and is a product of matrix algebras over $k$. Clearly $A \otimes_{k} B$ has the same form and it contains $S$.

Another suggestive consequence of the theorem is that when $k$ is algebraically closed, every algebraic element in a von Neumann regular $k$-algebra is contained in a finite-dimensional semisimple subalgebra. 
On the basis of the corollary, one might conjecture that (over an algebraically closed field) a finite-dimensional subalgebra of a regular algebra is contained in a finite-dimensional semisimple subalgebra. This is not the case, as the following example shows.

In general, suppose that $S$ is a finite-dimensional subalgebra of the regular algebra $R$ and $V$ is a left $R$-module. If, in addition, $S$ is contained in a finite-dimensional semisimple subalgebra $\bar{S}$, consider $V$ as a left $\bar{S}$-module. $V$ is a direct sum of simple $\bar{S}$-modules and there are only finitely many simple $\bar{S}$-modules available. Consequently $V$ is a direct sum of finite-dimensional indecomposable $S$-modules, with only finitely many isomorphism types appearing in the sum.

Now let $S$ be the three-dimensional algebra $k \cdot 1+k \cdot \alpha+k \cdot \beta$ where $\alpha^{2}=\beta^{2}$ $=\alpha \beta=\beta \alpha=0$. $S$ has finite-dimensional faithful indecomposable modules $V_{n}$ with $\operatorname{dim} V_{n} \geqslant n$ (cf. [2, p. 432]). Let $V=\sum_{n} V_{n}$ and set $R=\operatorname{Hom}_{k}(V, V) . R$ is von Neumann regular and $S$ imbeds in $R$ in the obvious fashion. If $S$ is contained in a semisimple subalgebra, as an $S$-module $V$ could also be written as a sum of indecomposables with bounded dimension. This contradicts a general vision of the Azumaya-Krull-Schmidt Theorem [1]. (Unfortunately, this choice of $R$ is not locally finite-dimensional. It is not easy to see how to adapt the example to answer Handelman's question.)

\section{REFERENCES}

1. F. W. Anderson and K. R. Fuller, Rings and categories of modules, Graduate Texts in Math., vol. 13, Springer-Verlag, New York, 1973.

2. C. W. Curtis and I. Reiner, Representation theory of finite groups and associative algebras, Interscience, New York, 1962.

3. K. R. Goodearl, von Neumann regular rings, Monographs and Studies in Math., vol. 4, Pitman, London, 1979.

4. N. Jacobson, Structure of rings, Amer. Math. Soc. Colloq. Publ., vol. 37, Amer. Math. Soc., Providence, R. I., 1964.

5. J. Levitzki, On the structure of algebraic algebras and related rings, Trans. Amer. Math. Soc. 74 (1953), 384-409.

Department of Mathematics, Virginia Polytechnic Institute and State University, BlacksBURG, VIRGINIA 24061 